\title{
EL MANEJO DE LA PÉRDIDA, EL TRAUMA Y EL PROCESO DE DUELO EN LA CONSEJERÍA A PERSONAS CON ABUSO DE SUBSTANCIAS
}

\author{
Manuel Antonio Rivera Acevedo ${ }^{1}$
}

\section{Resumen}

Contribuir en la reconstrucción de una identidad sana y una personalidad funcional conlleva desarrollar estrategias que favorezcan la resolución de eventos y situaciones que originaron y mantienen la conducta adictiva. Independientemente de las teorías usadas para explicar este tipo de conducta, las intervenciones profesionales y los tratamientos diseñados como parte del proceso de ayuda pueden enriquecerse incorporando el manejo de la pérdida, el trauma y el proceso de duelo en sus protocolos. De esta manera se favorecerá la reconstrucción de la identidad en la que la persona pueda proyectarse hacia un futuro de esperanza.

Descriptores: pérdida, trauma, duelo, proceso de duelo, discurso, proceso de ayuda.

\section{Abstract}

Aiding in the reconstruction of a healthy identity and a functional personality implies developing strategies that contribute to the resolution of events and situations that gave rise and maintain the addictive behavior. Regardless of the theories used to explain this type of conduct, professional interventions and treatments designed as part of the helping process could benefit by incorporating themes such as loss and trauma management, as well as the grieving process. This might enable the client in reconstructing his or her identity favoring a more hopeful future

Keywords: Loss, trauma, grief, grieving process, discourse, helping process. En camino hacia una nueva vida

Mirado localmente, el mundo de la adicción a las substancias controladas es un fenómeno de increíble alcance y difícil manejo. La adicción toca a personas, familias, comunidades y grupos sociales, interfiriendo adversamente con su organización interna, su funcionamiento y la dinámica de sus relaciones. Afecta la salud, el bienestar y la seguridad de cuantos se ven involucrados, ya sea por el consumo directo, por los 
efectos en otras personas o por el deterioro en sus relaciones. El fenómeno de la adicción tiene unas dimensiones económicas y políticas de suma complejidad, que a su vez influyen en todo intento por dar una respuesta adecuada y satisfactoria al mismo.

En el centro de todo, está la persona adicta, un ser humano que ha visto tronchada su historia como individuo y sobrevive como un ente fragmentado, con una identidad desintegrada y una personalidad al margen de la sociedad. Incapaz de narrarse a sí misma coherentemente y establecer relaciones satisfactorias, la persona que busca ayuda profesional para dejar atrás su conducta adictiva necesita asumir su pasado, responsabilizarse de su presente y delinear de una manera renovada su futuro. No es un proyecto fácil de plasmar en la realidad. En ocasiones implica desaprender el discurso utilizado hasta el momento, en el que su identidad pasa a un segundo plano y es vista como una persona enferma, una víctima, alguien sin fibra moral o como un criminal yaprender un nuevo lenguaje con el que pueda expresar sus más íntimas emociones, compartir sus sentimientos y recrearse como persona.

Brooks y McHenry (2009) postulan que es posible ayudar a la persona adicta en su deseo por salir del ciclo de la adicción y alcanzar una nueva vida. Es un proyecto que implica un gran esfuerzo y creatividad por parte del terapeuta y del cliente y abarca muchas áreas de atención. Según estos dos autores, uno de estos campos tiene que ver con la pérdida y el duelo como elementos del proceso de ayuda en el que se da inicio a un nuevo estilo de vida y de comportamiento. Estos dos conceptos, así como el de trauma, reconceptualizados para atender profesionalmente a la persona adicta posibilitan un nuevo enfoque terapéutico que puede enriquecer otros modelos y programas de ayuda.

El trabajo terapéutico a favor de la persona adicta conlleva una íntima colaboración entre el profesional y el cliente en el que los momentos neurálgicos del pasado y del presente puedan ser reinterpretados, quitándoles el poder que ejercen sobre la conducta actual. Desenmascarar dichos momentos, eventos o episodios implica reconocer la huella que han dejado en la vida y la dirección que han forzado a tomar, sea consciente o inconscientemente, de manera voluntaria o involuntaria. Este proceso, doloroso de por sí, puede hacerse un poco más manejable si en el diálogo promovido durante el proceso de ayuda se presenta el duelo como espacio en el que la pérdida y el trauma son incorporados como partes importantes en la reconstrucción de la identidad.

\section{La pérdida y el trauma}

En mayor o menor grado, todos hemos vivido el proceso de recuperación luego de una pérdida. Quedarse sin empleo o sin hogar, ver cómo los hijos se independizan y se marchan, perder movilidad física luego de una enfermedad o un accidente, dejar atrás la vitalidad de los años jóvenes, reconocer que las cosas no han salido como se esperaba, o experimentar la muerte de un ser querido, entre otros, son eventos que marcan 
profundamente a las personas. Si bien es cierto que la pérdida puede ser el momento para pasar a otra etapa de la vida y crecer como persona, también puede ser fuente de grandes incertidumbres, tristezas, sufrimientos y dolores. En algunas ocasiones, detiene momentáneamente la vida, mientras que en otras la descarrila totalmente. En ambas instancias, el resultado es un trauma, una experiencia que deja huellas en la manera en que la persona se ve a sí misma y a su mundo y que afecta sus relaciones, tanto consigo como con los demás.

Superar el trauma ocasionado por la pérdida, así como la pérdida misma, será más fácil o más difícil dependiendo del significado otorgado al objeto, la persona o la relación perdida. Si lo que se ha perdido era central en la vida, reanudar la marcha podría tomar más tiempo y esfuerzo que lo que se esperaría en otras circunstancias. Pero incluso si la pérdida fue de algo periférico o incidental, la recuperación podría ser de una magnitud sorprendente, particularmente si no le dio importancia al objeto o evento y ahora se descubre la centralidad del mismo. De acuerdo a la interpretación subjetiva dada al evento, la respuesta a la pérdida puede dar lugar a una experiencia traumática de gran complejidad, en la que la persona se siente incapaz de expresar sus sentimientos, manejar adecuadamente el pasado y plantearse positivamente el futuro (Ide Williams, 2006). De ahí que, tanto si es vista como algo positivo o negativo, superar la experiencia de la pérdida y el trauma asociado a ella conlleva nuevos aprendizajes y estilos de conducta, los cuales no siempre son fáciles de adquirir, mantener y desarrollar.

\section{El proceso de duelo}

Para responder adaptativamente a todo tipo de pérdida, es preciso pasar por el duelo, un proceso de ajuste que se produce como reacción a lo ocurrido (Humphrey, 2009). El duelo proporciona un tiempo y un espacio más o menos seguro en el cual se pueden expresar los sentimientos, aceptar el carácter irreversible y en ocasiones irreparable del evento, afrontar los cambios en uno mismo y en las relaciones con otras personas y hacer las modificaciones y adaptaciones necesarias para seguir adelante. Durante el proceso de duelo será imprescindible manejar emociones, en ocasiones aparentemente contradictorias entre sí, como la tristeza, la ansiedad, el coraje, la soledad, la angustia y la desesperanza. Muy probablemente, será necesario responder también a momentos de gran confusión en los que la persona se siente aislada de otras y experimenta un estado de aturdimiento, tanto cognitivo como afectivo. Sorprendentemente, en algunas ocasiones se verá forzada a reaccionar a sentimientos de alivio y hasta de euforia momentánea que pueden hacerle sentir despreciable ante ella misma. Es debido a la complejidad del proceso que el duelo, como respuesta a la pérdida, puede ser en sí mismo una experiencia tan traumática como la pérdida misma. De su manejo dependerá el crecimiento o el estancamiento de la persona. 
El duelo guarda relación con la manera en que otorgamos sentido y organizamos los recuerdos que hacen referencia al evento perdido. No es tarea fácil. Según Janet (citado en Ide Williams, 2006), la respuesta a un evento o experiencia traumática, como las que se producen como resultado a una pérdida, encuentran resonancia en la salud de la persona, y pueden dar lugar a conductas patológicas. Ide Williams indica que Janet llamó a estas respuestas emociones vehementes, postulando que tienen su origen en el estado de la persona y en la interpretación cognitiva que hace de la situación vivida. En su artículo, Ide Williams expresa que el trauma teje su propia historia, una en la que el sufrimiento fragmenta la vida interior y los mecanismos de defensa, tanto físicos como emocionales, de la persona. Si la naturaleza del trauma es de carácter repetitivo o acumulativo y se hace imposible reanudar la vida, el resultado será conductas complejas y persistentes que afectarán los sistemas biológicos, psicológicos y sociales de la persona, impidiéndole retomar sanamente la misma.

El proceso de duelo tiene que ver con los recuerdos, y el manejo de los mismos es sumamente complicado. Citando a Morrison (1987) y a Greenberg (1998), Ide Williams (2009) hace referencia al re-recuerdo (rememory), un recuerdo que se niega a desaparecer, se adueña del superviviente y se reorganiza de manera confusa fuera de los límites del tiempo. Cuando reaparece, confunde la realidad presente con la pasada y la persona se siente incapaz de hacer sentido de sí misma y de su vida. En otras palabras, comienza a disfuncionar, porque el modelo interno que tenía de sí misma, así como el modelo que tenía de su mundo dejan de servirle como antes. Sin un sentido de sí misma, en el que se percibe como amable por otros, la persona deja de regular sus estados internos y se aferra a cualquier tipo de vínculo que le proporciona una sensación de estabilidad y seguridad. Este tipo de conducta se explica de manera excelente por los postulados de la Teoría de la Relaciones Objetales, en la que se identifican objetos o parte de objetos que sirven para satisfacer necesidades personales entendidas como básicas y proveen una sensación de seguridad y de placer. En este caso, el recuerdo mismo, convertido en memoria a través del significado conferido, puede convertirse en el objeto que impide u obstaculiza el crecimiento de la persona.

Finalmente, el duelo es expresado mediante el lenguaje y otros símbolos creados socialmente. No obstante, la magnitud o importancia de la pérdida puede ser tal que la persona se siente incapaz de expresar verbalmente o por otros medios cuanto acontece en su interior, sintiéndose en el más profundo aislamiento. Negada, aunque sea momentáneamente, de la posibilidad de relacionarse con otros y hacerles compartir su experiencia, la persona afectada puede sentirse frente a un abismo profundo, sin esperanza alguna. De ser así, el duelo, a pesar de su dimensión catártica, no contribuiría al manejo de la pérdida y a la resolución de trauma, complicando el proceso de recuperación. 


\section{La pérdida y el duelo en la adicción a substancias controladas}

Haberstroh (2005) postula que la pérdida es la nota distintiva de la adicción. Según este autor, la conducta adictiva resulta muchas veces en pérdidas que se manifiestan como marcadas disminuciones en el funcionamiento físico, relacional y psicológico. Conforme van poniendo en jaque sus creencias y valores personales, las personas adictas experimentan rechazo por parte de los sistemas de apoyo social. Las múltiples pérdidas experimentadas abarcan tanto lo tangible como lo intangible. Más aún, se arriesgan a perder el sentido de identidad como individuos y la sensación de continuidad en la vida, afectando adversamente el desarrollo de la misma.

Al principio, insensibilizadas emocionalmente por el uso de la substancia adictiva, el sentido de pérdida es mínimo (Haberstroh, 2005). Corresponde al estado de precontemplación en el Modelo Transteórico postulado por Prochaska, Di Clemente y Norcross (1992), en el que la persona ni siquiera da cabida a la posibilidad de tener un problema y defiende su conducta alegando que no le hace daño a nadie y puede controlarla en el momento en que lo desee.

Haberstroh (2005) identifica pérdidas que anteceden a la adicción y pueden haber contribuido a la misma, pérdidas asociadas a la adicción y pérdidas asociadas al tratamiento. Entre las pérdidas antecedentes hace referencia a las condiciones en el hogar, las relaciones de cercanía o intimidad, la posibilidad de haber sido objeto de cualquier tipo de abuso y no haber manejado adecuadamente el mismo, así como factores asociados con la falta de oportunidades para el éxito en la vida y la posibilidad de alcanzar la actualización como persona. Las pérdidas asociadas a la adicción, a las que ya se ha hecho referencia, incluyen las relacionadas con la salud, la familia, el trabajo, los bienes materiales, la imagen de sí mismo y las relaciones con personas significativas. Finalmente, las pérdidas relacionadas con el tratamiento, tal vez las más significativas en el contexto del proceso de ayuda, guardan relación con la aceptación de la responsabilidad personal por las consecuencias de la conducta adictiva y la construcción de un futuro radicalmente distinto a lo vivido hasta el presente.

Es precisamente en el manejo terapéutico de este último grupo de pérdidas en las que el proceso de duelo desempeña un papel de gran importancia. Conforme la persona adicta va saliendo del letargo emocional, cognitivo y afectivo en el que ha estado sumida, se va dando cuenta de los múltiples daños provocados, tanto a sí misma como a otros. Ahora bien, junto a la oportunidad de asumir responsabilidad por conductas pasadas, las expectativas frustradas, la confianza traicionada, las oportunidades malogradas, pueden resurgir con gran virulencia, obstaculizando o descarrilando el proceso de recuperación. Experimentar de manera vivencial un proceso de duelo puede ser el elemento clave que decida el éxito o el fracaso de la empresa mediante la cual la persona adictiva intenta reconstruirse. 
En su artículo acerca del trauma complejo, Ide Williams (2006) hace referencia al mito de Dionisio, cuyo cuerpo es desmembrado y esparcido a lo largo de la tierra. Salir de la conducta adictiva conlleva identificar y asumir los eventos que dieron lugar al desmembramiento físico, psicológico y social, simbolizados por la destrucción de la integridad personal, aceptar el grado de responsabilidad que pueda atribuirse en cada caso y procurar re-membrar correctamente la vida dañada o distorsionada. En el marco del escenario terapéutico, correspondiente a las etapas de contemplación y preparación para el cambio identificadas por Prochaska, Di Clemente y Norcross (1992), este proceso de reintegración y reconstrucción puede dar lugar a emociones vehementes que van desde la vergüenza hasta el terror por lo vivido, las que pueden desembocar en un sentimiento de incapacidad ante los retos que conlleva la reconstrucción de la vida. Es por eso que el manejo adecuado de estas emociones, sentimientos y cogniciones debe atender a las posibles resistencias al cambio por parte de la persona adicta, muchas veces producto de un miedo indescriptible ante lo que ha transcurrido y ante lo que está por venir.

La persona adicta que contempla salir de su conducta maladaptativa no sólo tiene que asumir su pasado, algo traumático en sí mismo, sino que además debe orientarse hacia el futuro, incierto en el mejor de los casos, terrible cuando se cree haber perdido toda esperanza. Por la gravedad del asunto, puede resistirse a todo intento de cambio. Esta resistencia, identificada por Miller y Rollnick (2002) como parte esencial del proceso de ayuda orientado al cambio, puede ser vencida mediante la construcción de una nueva narrativa personal mediante la cual, según Hunt y McHale (2008), la persona adicta puede desarrollar una visión coherente de su pasado, integrarlo con el presente e identificar las maneras en las que intentará responder a los retos del futuro. Éste es precisamente el proceso de duelo mediante el cual se asumen las pérdidas vividas, se les da nuevo significado y se proyecta una nueva identidad personal.

\section{El proceso de ayuda}

Al hablar acerca del proceso de transformación, Widderschoven (citado en Ide Williams, 2006), menciona que el cambio en la manera de ver el mundo no es una empresa teórica alcanzable por un individuo, sino un proceso práctico que surge de la interacción y el diálogo. Mediante el intercambio que se produce a lo largo del proceso de ayuda, el terapeuta y el cliente podrán identificar cómo la conducta adictiva se convirtió en un discurso con el cual el cliente intentaba expresar algo, tal vez no de la manera más creativa posible, pero que terminó limitando su funcionalidad y anulando toda posibilidad de crecimiento y desarrollo óptimo de las potencialidades individuales.

En el diálogo terapéutico se debe dar oportunidad para identificar y expresar los sentimientos que tienen que ver con experiencias positivas y negativas. Precisamente por la naturaleza de estos sentimientos, es importante evitar que este proceso se malinterprete como uno de fácil desarrollo. A tales efectos, Wortman (citada en Boerner, 
Wang y Cimarolli, 2006) advierte que la expectativa de cambios positivos puede obstaculizar y hasta impedir el proceso de afrontamiento. En la reconstrucción de la persona no son recomendables los atrechos ya que, al no dejar definitivamente zanjadas situaciones medulares al proceso, puede contribuir a que las mismas resurjan con mayor fuerza en el futuro y descarrilen todo intento orientado a la salud y al bienestar del cliente.

En el campo de la ayuda profesional, para dejar atrás la conducta adictiva, este nuevo diálogo conlleva reformular las pérdidas como déficits adquiridos en la funcionalidad del individuo. En efecto, la conducta adictiva puede darse en la disminución o ausencia de relaciones significativas así como en la imposibilidad de fijar metas y diseñar estrategias para alcanzar las mismas. La persona adicta deja de cuidarse a sí misma y llega a adoptar un discurso que muchas veces la aliena de los demás en vez de crear vínculos que le provean un anclaje emocional y cognitivo en su vida.

En su estudio sobre el impacto sobre la pérdida funcional en personas con limitaciones visuales adquiridas, Boerner, Wang y Cimarolli (2006) presentan una serie de ideas que podrían ser incorporadas en el proceso de ayuda al cliente que desea superar su conducta adictiva. En el caso de los participantes investigados, los autores hacen referencia a la importancia de reconocer lo irreversible de la pérdida de la visión, ya sea a causa de una enfermedad o de un accidente. Dicha pérdida puede dar lugar a limitaciones significativas y a un disminuido sentido de competencia personal. Esto, a su vez, puede contribuir a sentimientos de coraje, miedo y hasta culpa, llevando en ocasiones a que la persona afectada cuestiones su lugar y su propósito en la vida.

Manejados adecuadamente, Boerner, Wang y Cimarolli (2006) señalan que todos estos sentimientos y experiencias traumáticas pueden contribuir a un nuevo posicionamiento en la vida, en el que se establecen y reordenan nuevas prioridades a la vez que se adquieren destrezas que permiten afrontar y manejar los múltiples vaivenes que se darán en el futuro. Los autores insisten en que no se puede ignorar los cambios negativos ni la influencia de los mismos ya que están tejidos en la vida del cliente. No obstante, asumidos y valorados correctamente, permiten evaluar la vida desde una nueva perspectiva y darle un nuevo significado al contenido y al contexto del cambio. En el escenario descrito, las ideas asociadas con la pérdida, el trauma y el duelo encuentran una ejemplificación y aplicación práctica excelentes que pueden ser llevadas al proceso de ayuda con personas que desean salir de la adicción.

En la ayuda terapéutica a la persona adicta, las ideas postuladas por Boerner, Wang y Cimarolli (2006) encuentran eco en las señaladas por Etherington (2007). No importa la teoría usada para explicar la conducta adictiva, esta autora resalta la importancia de que los profesionales de ayuda aprendan a escuchar de manera diferente las historias de trauma y pérdida de sus clientes, ayudándoles a adquirir un agarre orientado a la reflexión que les permita recontar lo vivido sin arriesgarse a caer en nuevas experiencias 
traumáticas. El marco terapéutico delineado por DiClemente (2003), en el que se hace el mismo recorrido desde la contemplación hasta la acción que se sigue para diseñar el proceso de ayuda sirve para determinar cuál fue el origen de la conducta adictiva. Permite que el cliente identifique asuntos y eventos que contribuyeron a su estado actual y pueda comenzar a responder adecuadamente a los mismos.

El diálogo entre terapeuta y cliente debe favorecer que la historia contada pueda ser reinterpretada y reconstruida según se va narrando. En ocasiones, faltarán las palabras para hacerlo. Etherington (2007) cita a Bruner (1986), quien dijo que sólo conocemos aquello para lo que tenemos un lenguaje que nos permite conocer. Cuando no hay lenguaje o marco de referencia, no es posible entender lo que está pasando y si no se entiende, no se puede expresar. El trabajo de reinterpretación y reconstrucción de la narrativa personal con personas adictas exige reconocer lo limitado del lenguaje y del discurso social con los cuales la persona adicta ha vivido hasta el momento. Es un lenguaje de deprivación a la vez que de sensaciones equivocadas acerca del placer y el bienestar. Al mismo tiempo, es un lenguaje de falsas expectativas y de enormes consecuencias, por lo que se hace imprescindible proveer herramientas que permitan una expresión más exacta de las emociones y los sentimientos a fin de que la persona sienta que el cambio no sólo es deseable, sino posible.

El propósito principal de este diálogo terapéutico es ayudar al cliente a adueñarse de su pasado, integrarlo al presente y proyectarlo a un futuro renovado. Este proceso conlleva identificar correctamente los recuerdos y a ordenarlos según su significado e importancia. A tales efectos, Hunt y McHale (2008) también citan a Janet (1925), al postular la existencia de la memoria traumática, en la que el individuo repite inconscientemente el pasado y es incapaz de salir del mismo, y la memoria narrativa, en la que el individuo narra el pasado como pasado, asumiéndolo y dándole un nuevo significado. Alcanzar este segundo estadio debe ser la meta de toda acción terapéutica.

Wrye (citado en Hunt y McHale, 2008) advirtió que las narrativas personales no se limitan a contar lo pasado, sino que son los medios por los cuales se forja y desarrolla la identidad. Estas narrativas están formadas por experiencias a las que se les ha otorgado algún tipo de significado y que en el proceso terapéutico son traídas a la memoria para intentar explicar o justificar la conducta. Bartlett (citado en Hunt y McHale, 2008) señala que la memoria es una construcción sujeta a muchas influencias y, por lo mismo, no tiene que ser exacta. El profesional de ayuda deberá estar atento a la manera en que el cliente da importancia a los eventos rememorados, la singularidad de los mismos y la elaboración imaginativa con que los integra en su narración. Este autor hace referencia al concepto de "confabulation", constructo para el cual no hay una traducción satisfactoria en español. El mismo hace referencia al olvidar o recordar selectivos que permite a la persona construir una historia de cuya veracidad está plenamente convencida. Las historias narradas por las personas adictas están marcadas notablemente por este tipo de ejercicio mental, por lo que deberán ser atendidas adecuadamente a fin 
de que el proceso de recuperación sea lo más efectivo posible.

Es precisamente aquí donde puede darse un proceso de duelo verdaderamente terapéutico. Ayudar al cliente a identificar los elementos que contribuyeron a sus múltiples pérdidas y proveerle un lenguaje mediante el cual pueda expresar sus sentimientos con respecto a las mismas equivale a proveerle un conocimiento mediante el cual pueda adquirir poder para obrar en su favor. Según McComarck (citado en Etherington, (2007), este conocimiento será siempre algo situado, transitorio, provisional y forzosamente parcial, pero no obstante, es una herramienta da gran valor para el diálogo terapéutico. Según Etherington, será un diálogo en el que se escucharán muchas voces, verdades y significados, en ocasiones contradictorias entre sí, pero que permitirán recontar, recuperar y restaurar a la persona que durante largo tiempo ha sido una desconocida para sí misma.

Conforme se desarrolla el proceso de ayuda, se va dando lugar a la identificación de las pérdidas y al duelo por las mismas. Es esencial no minimizar los sentimientos expresados por el cliente y permitir que el sentido de responsabilidad hacia lo vivido se vaya recuperando o adquiriendo poco a poco. En inglés hay dos vocablos: knowledge y acknowledge que explican esta parte del proceso. No basta con adquirir conocimiento (knowledge) de lo vivido. Para poder trascenderlo, hasta falta tomar conciencia del mismo (acknowledge) y aceptar las consecuencias de las experiencias vividas. Es aquí donde comienza un duelo que puede desgarrar internamente a la persona, pero el cual es preciso atravesar para poder pasar a la siguiente etapa de reconstrucción de la identidad.

\section{Restitución y retribución}

En su trabajo sobre el trauma y la recuperación, Herman (1992) hace referencia a la necesaria restitución de la persona que ha sido impactada por una experiencia traumática. Describe la restitución como la restauración de un sentido de eficacia y de poder que permiten recuperar las facultades psicológicas de confianza, autonomía, iniciativa, competencia, identidad e intimidad. En efecto, el evento traumático ha privado a la persona del ejercicio de dichas facultades, llevándola incluso a creer que están enteramente vedadas para ella. Contribuir en la restitución de las mismas es un paso de vital importancia en la reconstrucción de la persona y en la formulación de oportunidades y experiencias distintas a las vividas.

No obstante, a juicio del que suscribe, en el caso de la persona adicta que busca tanto una nueva conducta como una nueva vida, la restitución es tan sólo un primer paso en el camino hacia la recuperación. Es vital añadir el aspecto de retribución. Conforme vaya recuperando el uso de las facultades arriba mencionadas, la persona adicta se verá en la obligación de plantearse el grado de responsabilidad ante lo vivido. No sólo 
ha tronchado su propia existencia, sino que ha trastocado la vida de sus familiares, amigos y personas significativas. Ahora bien, reconocer que ha sido así no es suficiente. Para que el proceso de rehabilitación se complete, es necesario retribuir de alguna manera a las personas o grupos puedan haberse visto afectados por el comportamiento adictivo en sus múltiples manifestaciones, sin olvidar al individuo mismo, víctima primera de sus decisiones. Sólo así el duelo por la pérdida experimentada y la recuperación de los traumas subsiguientes tendrá una verdadera oportunidad de éxito.

\section{Conclusión}

El trabajo de consejería y ayuda terapéutica con personas que manifiestan una conducta adictiva es arduo y agotador. Implica ir al encuentro de un ser humano que se siente privado de esa misma humanidad que le da derecho a relacionarse con otras personas y a construir su vida. Este encuentro no está exento de contradicciones, altos y bajos, logros y fracasos. El discurso social, aprendido y asimilado por la persona adicta, puede distanciarla de todo intento de mejoría que se le presente.

Por otro lado, si la persona toma la decisión de romper con su pasado, recuperar su identidad y reconstruir su personalidad, necesitará de un acompañamiento y una ayuda que le permitan solucionar conflictos y eventos pasados que arrastra e influyen en su conducta presente. En la mayoría de estos asuntos estará presente el fenómeno de la pérdida, ya sea como resultado directo o indirecto de la conducta adictiva y la necesidad de responsabilizarse por las acciones que hayan contribuido a la misma. Este proceso conlleva atravesar un período de duelo mediante el cual la persona pueda adquirir un nuevo lenguaje y un nuevo discurso que le permita expresarse tal cual es, sanar sus heridas y adentrarse en el futuro.

Por parte del profesional de ayuda, es importante recordar que en todo diálogo todas las personas involucradas se ven afectadas. A tales efectos, Bober, Regher y Zhou (2006) advierten acerca de la importancia que tiene el cuidado personal por parte del profesional de ayuda. Exponerse diariamente a historias que tienen que ver con pérdida y trauma prueba los recursos internos del terapeuta. Iniciar y proseguir un proceso de duelo en el que el cliente se enfrenta a los aspectos más sombríos de su persona, intentando salir a la luz, puede arrojar sombras en la vida del terapeuta. De ahí la importancia de desarrollar sus propias estrategias de afrontamiento y manejo, a fin de que su intervención en estas iniciativas le permitan consolidar sus logros como profesional y crecer como persona. 


\section{Referencias}

Bober, Ted, Regher, Cheryl, \& Zhou, Yanqui. (2006). Development of the Coping Strategies Inventory for trauma counselors. Journal of Loss and Trauma, 11, 71-83.

Boerner, Kathrin, Wang, Shue-Wen, \& Cimarolli, Verena. (2006). The impact of functional loss: Nature and implications of life changes. Journal of Loss and Trauma, 11, 265-287.

Brooks, Fred, \& McHenry, Bill. (2009). A contemporary approach to substance abuse and addiction counseling: A counselor's guide to application and understanding. Alexandria, VA: American Counseling Association.

Bruner, Jerome.(1986). Actual minds, possible worlds. Cambridge, MA: Harvard University Press.

DiClemente, Carlo. (2003). Addiction and change: How addictions develop and addicted people recover. New York: The Guilford Press.

Etherington, Kim. (2007). Loss and trauma in the lives of people with a history of drug abuse. Journal of Loss and Trauma, 12, 59-72.

Frank, A. W. (2000). The standpoint of storyteller. Qualitative Health Research, 10, 354-365.

Haberstroh, S. (2005). Facing the music: Creative and experiential group strategies for working with addiction related grief and loss. Journal of Creativity in Mental Health, 1(3/4), 41-55.

Herman, Judith. (1992). Trauma and recovery: The aftermath of violence - from domestic abuse to political terror. NY: Basic Books.

Humphrey, Keren. (2009). Counseling strategies for loss and grief. Alexandria, VA: American Counseling Association.

Hunt, Nigel, \& McHale, Sue. (2008). Memory and meaning: Individual and social aspects of memory narratives. Journal of Los and Trauma, 13, 42-58.

Ide Williams, Wendy. (2006). Complex trauma: Approaches to theory and treatment. Journal of Loss and Trauma, 11, 321-335.

Janet, Pierre. (1925). Psychological Healings, Vols. 1 \& 2. London: Macmillan. 
McCormak, C. (2004). Storying stories: A narrative approach to in-depth interview conversations. International Journal of Social Research Methodology, 7, 219236.

Miller, William, \& Rollnick, Stephen. (2002). Motivational Interviewing: Preparing people for change ( $2^{\text {nd }}$ ed.). New York: The Guilford Press.

Prochaska, James, DiClemente, Carlo, \& Norcross, John. (1992). In search of how people change $\square$ :Applications to the addictive behaviors. American Psychologist, 47, 1102-1114.

Widderschoven, G. A. (1999). Cognitive psychology and hermeneutics: Two approaches to meaning and mental disorder. Philosophy, Psychiatry \& Psychology, 6, 245-253.

Wrye, H. K. (1994). Narrative scripts: Composing a life with ambition and desire. American Journal of Psychoanalysis, 54, 127-141. 